\title{
Good versus Moderate Regulation of Alloxan-induced Diabetes in Arteriosclerotic and Nonarteriosclerotic Rats
}

\author{
B. C. Wexler and D. G. Sangrey \\ The May Institute for Medical Research of the Jewish Hospital Association and the Departments of Medicine and Pathology, \\ University of Cincinnati College of Medicine, Cincinnati, Ohio, USA
}

Summary. Nonarteriosclerotic (virgin) and arteriosclerotic (breeder) rats were made diabetic with alloxan. Animals were treated with constantly adjusted doses of insulin, occasionally adjusted doses, or no treatment. The obese breeder rats lost weight; the lean virgin rats gained weight. All of the animals were autopsied 5 months post-alloxan. Blood pressure increased in virgin rats; the pre-existent mildly elevated blood pressure of arteriosclerotic breeder rats decreased. Glucosuria was effectively reduced in animals accorded good control; blood glucose was not effectively controlled. Circulating nonesterified fatty acids and triglycerides were more effectively normalised by insulin therapy. The diabetes was accompanied by adrenal hypertrophy, thymus gland involution, and increased circulating corticosterone. Virgin rats $(68 \%)$ developed arterial disease when made alloxan-diabetic; treatment with insulin completely prevented arterial disease. Breeder rats with pre-existent arteriosclerosis showed worsening of their cardiovascular disease; when made severely diabetic those provided with good control of their diabetes were protected against exacerbation of their vascular disease.

Key words: Alloxan, coronary arteries, diabetes, hyperglycaemia, insulin, intimal calcification, nonesterified fatty acids, Sprague-Dawley rats, total cholesterol, triglycerides.

Repeatedly bred, male and female rats will develop hyperglycaemia, hyperlipidaemia, hypertension, arteriosclerosis, and age prematurely [1-4]. Virgin rats do not develop any of these degenerative changes spontaneously until they are 3 years old [5].

We have produced alloxan diabetes in nonarteriosclerotic (virgin) and arteriosclerotic (breeder) rats and withheld treatment. These animals developed severe ketosis, hyperglycaemia, hyperlipidaemia, and a fatty liver, the pre-existent arteriosclerosis of the breeder rats became severely exacerbated, and the formerly healthy virgin rats also developed arteriosclerosis [6-8]. In a similar experiment, hypophysectomy caused a definite improvement in the metabolic status of both the alloxandiabetic arteriosclerotic and non-arteriosclerotic animals, including a reduction in hyperglycaemia and hyperlipidaemia, but the arteriosclerosis of the breeder rats became inexorably worse [9].

In this experiment, we subjected nonarteriosclerotic virgin and arteriosclerotic breeder rats to alloxan diabetes. Some animals were given insulin to maintain urinary glucose at decreased levels, some animals were given insulin with constant adjustment of the dose to maintain optimum body weight and urine free of glucose, or no treatment. Our purpose was to determine whether improvement in the metabolic derangements which accompany diabetes would bear any relationship to the pathogenesis of arterial disease.

\section{Materials and Methods}

Previous experience with virgin and breeder rats made severely diabetic with alloxan [6-9] necessitated the provision of a large number of animals $(\mathrm{n}=$ 500 ) at the outset to allow for the great number of deaths anticipated during the first 7 days of alloxaninduced ketosis and unrelenting diabetes. Adult, male and female, virgin rats were used as subjects having no pre-existing arteriosclerosis. Male and female, repeatedly bred rats were used as subjects with pre-existing mild hyperglycaemia and arteriosclerosis. All were Sprague-Dawley rats between 6 and 9 months of age. The female breeders had com- 
pleted 4 or 5 successive pregnancies commencing when they were 90 days of age. These female breeders consistently display moderate to severe, grossly visible arteriosclerosis (see below). This latter group served as subjects with advanced, pre-existing arterial disease. The nonarteriosclerotic, male and female, virgin rats were raised under the same conditions of diet, light, temperature and humidity control as the arteriosclerotic breeder rats. When the female breeders had been weaned of their young after their fourth or fifth pregnancy, all of the animals were transferred to individual cages and were subjected to an $18 \mathrm{~h}$ fast prior to the injection of alloxan. Four groups of male and female, nonarteriosclerotic virgin rats, and male and female arteriosclerotic breeder rats ( $\mathrm{n}=24$ per group) served as non-treated, baseline controls. The remaining 452 animals consisting of equal numbers of male and female virgins and male and female breeders were then subjected to alloxan-induced diabetes.

A single SC injection of alloxan (Eastman Kodak) $10 \mathrm{mg} / 100 \mathrm{~g}$ body weight was given to both the arteriosclerotic and nonarteriosclerotic rats. Insulin therapy was withheld from some animals. The dose of NPH insulin (Lilly), 0.3-0.9 U/100 $\mathrm{g}$ body weight, was adjusted according to daily weight, and urinary glucose and protein (Labstix) at 0700 and $1700 \mathrm{~h}$. This formed the "good control" group. In the "moderate control" group, changes in body weight or the appearance of glucose or protein in the urine were not carefully regulated. Throughout the five month course of this experiment, the animals were fed a regular commercial rat chow (Purina) which was relatively low in fat (4\%). Blood (tail vein) was taken from representative animals $(n=6)$ from each group on Days 3 and 7 post-alloxan and after 1, 2, 3, 4 and 5 months ( $\mathrm{n}=10$ to 24 ) to correlate urinary with blood glucose levels.

Prior to autopsy, the systolic blood pressure of each animal was recorded using the Friedman: Freed, indirect, microphonic manometer and tail cuff apparatus; each of the animals was anaesthised lightly with $2.5 \mathrm{mg} / 100 \mathrm{~g}$ body weight quinalbarbitone (Seconal, Lilly) IP, and blood withdrawn by heparinised syringe from the abdominal aorta. The blood was spun in a refrigerated centrifuge and the serum frozen and stored until analysis. Automated techniques (Auto-analyzer: Technicon) were used to measure blood glucose, triglycerides, non-esterified fatty acids and total cholesterol. Serum corticosterone, the main adrenocortical steroid produced by the rat, was measured by an automated fluorometric method [10].

The heart and main arteries of each animal were examined carefully for any grossly visible evidence of vascular disease. Male breeder rats develop microscopic lesions of the aorta which cannot be detected by gross inspection; female breeder rats develop grossly visible lesions of the aorta which first become visible in the abdominal aorta as early as their second or third pregnancy [1-4]. As the aortic lesions worsen, they appear next in the aortic arch and thoracic aortic segments. The heart and aorta of each animal was examined carefully at autopsy. The severity of cardiovascular disease in male breeders is established by subsequent histopathologic examination; in female breeders, quantitative assessment may be made at autopsy. Details of the quantification of aortic lesions in breeder rats have been published [1]. Pertinent organs from each animal were trimmed and weighed and then fixed in neutral formalin for histopathologic examination. In addition to haematoxylin and eosin, special stains were used to demonstrate metachromasia and mucopolysaccharides (alcian and toluidine blue, Hale stain), elastic tissue (Verhoeff-Van Gieson), calcium (von Kossa), B cell granularity of the islets of Langerhans (aldehyde fuchsin-Ponceau), and lipids in frozen sections (oil red $O$ and sudan black $B$ ).

The gravimetric and biochemical data were subjected to statistical analysis by analysis of variance or Student's t-test [11]. $P$ values greater than 0.05 were considered to be not significant.

\section{Results}

\section{General Observations}

Within $2 \mathrm{~h}$ of the single injection of alloxan, all animals showed marked glucosuria. Within $24 \mathrm{~h}$, all of the animals were hyperglycaemic and hyperlipidaemic. Polyphagia, polydypsia, and polyuria were present. Mortality was high, reaching a maximum on the 4th day post alloxan. Those animals which died at this time had a severely fatty liver at autopsy. During the acute post-alloxan hyperglycaemia, relatively mild doses of insulin, $0.3-0.9 \mathrm{U}$, frequently led to death of the animal. Paradoxically, these deaths were due to hypo- or hyperglycaemia. At no time would these animals tolerate a single, incremental increase or reduction in insulin greater than $0.3 \mathrm{U}$.

\section{Weight Changes}

Arteriosclerotic breeder rats are characteristically obese compared to their healthy virgin counterparts (Table 1). When these arteriosclerotic animals were made severely diabetic, they lost a considerable 
Table 1. Changes in blood pressure, and body and organ weights of male and female, virgin and breeder, Sprague-Dawley rats subjected to alloxan-induced diabetes and autopsied 5 months post-alloxan

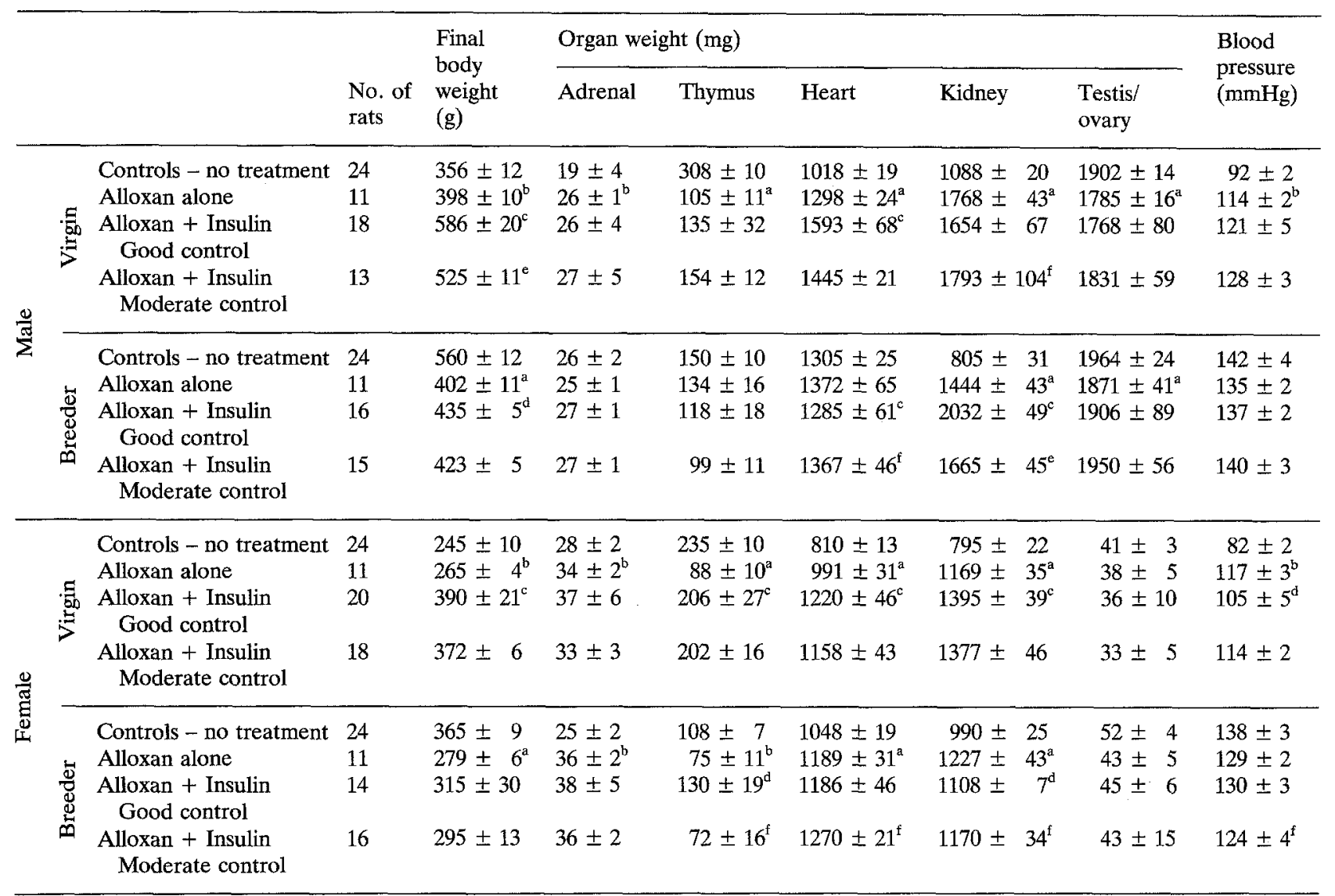

All values cited are the Mean $\pm \mathrm{SE}$

${ }^{\mathrm{a}} \mathrm{p}<0.001,{ }^{\mathrm{b}} \mathrm{p}<0.01$, compared to no treatment

${ }^{\mathrm{c}} \mathrm{p}<0.001,{ }_{\mathrm{d}}^{\mathrm{d}} \mathrm{p}<0.01$, compared to treatment with alloxan alone

${ }^{\mathrm{e}} \mathrm{p}<0.001,{ }^{\mathrm{f}} \mathrm{p}<0.01$, compared to alloxan + insulin or good control

Only one of paired organs was weighed

amount of weight. Treatment with insulin helped to ameliorate this severe weight loss (Table 1). Arteriosclerotic male breeder rats showed the most severe weight loss after being made diabetic. In direct contrast, the virgin rats became heavier when made diabetic; treatment with insulin increased their diabetes-induced obesity (Table 1 ).

The diabetic animals manifested considerable adrenal hypertrophy concomitant with severe thymus gland involution (Table 1). The hearts and kidneys of the diabetic animals were greatly enlarged and the testes and ovaries were significantly reduced in size and weight (Table 1).

\section{Systolic Blood Pressure}

The blood pressures of the nonarteriosclerotic virgin rats increased after the induction of diabetes, treated or non-treated (Table 1). In contrast, the blood pressure of the arteriosclerotic breeder rats showed no significant change with the induction of diabetes irrespective of treatment with insulin with the exception of the female breeders (Table 1).

\section{Blood Chemistry}

\section{Serum Glucose}

During the first few weeks, all of the animals were severely hyperglycaemic (Figs. 1) and ketotic. The severe hyperglycaemia and ketosis subsided gradually. Two months post-alloxan, the blood glucose level ranged between 600 and $800 \mathrm{mg} / 100 \mathrm{ml}$ in untreated rats but remained at about $600 \mathrm{mg} / 100 \mathrm{ml}$ during the remaining 3 months until the close of the experiment (Fig. 1). Although the arteriosclerotic animals (male and female) were mildly diabetic 


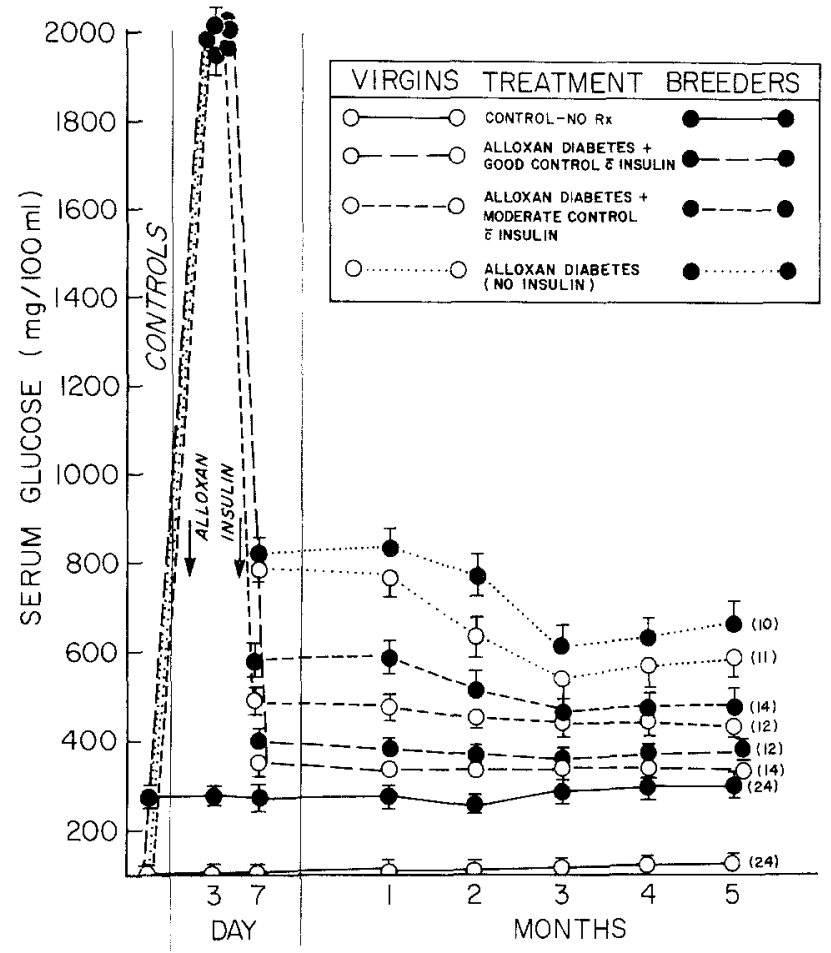

Fig. 1. Comparison of the blood glucose levels of male, nonarteriosclerotic virgin rats and breeder rats with pre-existing arteriosclerosis subjected to 5 months of alloxan-induced diabetes. Virgin and breeder rats which received no alloxan served as controls. Some animals were subjected to alloxan-diabetes with no insulin therapy. Some received insulin twice daily with doses regulated carefully according to their ability to maintain body weight and urine free of sugar and protein, "good control". Others were deliberately permitted to show some sugar in their urine and to lose body weight, and insulin injections were omitted occasionally, "moderate control". Blood glucose was estimated $(\mathrm{n}=6)$ in each group on days 3 and 7, and at 1,2,3 and 4 months after alloxan. All of the animals were autopsied at 5 months after alloxan $(\mathrm{n}=$ number of surviving animais). Blood glucose levels in females (virgins and breeders) were not statistically significantly different from males by analysis of variance. Each point $=$ mean \pm SEM

(serum glucose $245 \pm 10 \mathrm{mg} / 100 \mathrm{ml}$ and $220 \pm$ $9 \mathrm{mg} / 100 \mathrm{ml}$, respectively) prior to the injection of alloxan, all of the animals exhibited a similar degree of hyperglycaemia in response to the injection of alloxan (Fig. 1). Despite virtually complete clearance of glucose from the urine of animals provided good control with insulin, their blood glucose levels remained relatively high, $325-375 \mathrm{mg} / 100 \mathrm{ml}$ (Fig. 1).

\section{Serum Triglycerides}

Arteriosclerotic male and female breeder rats were hyperlipidaemic (Figs. 2, 3, 4) compared to their nonarteriosclerotic, virgin counterparts. The alloxaninduced diabetes caused severe hypertriglyceridaemia in all animals (Fig. 2). The regimen of good

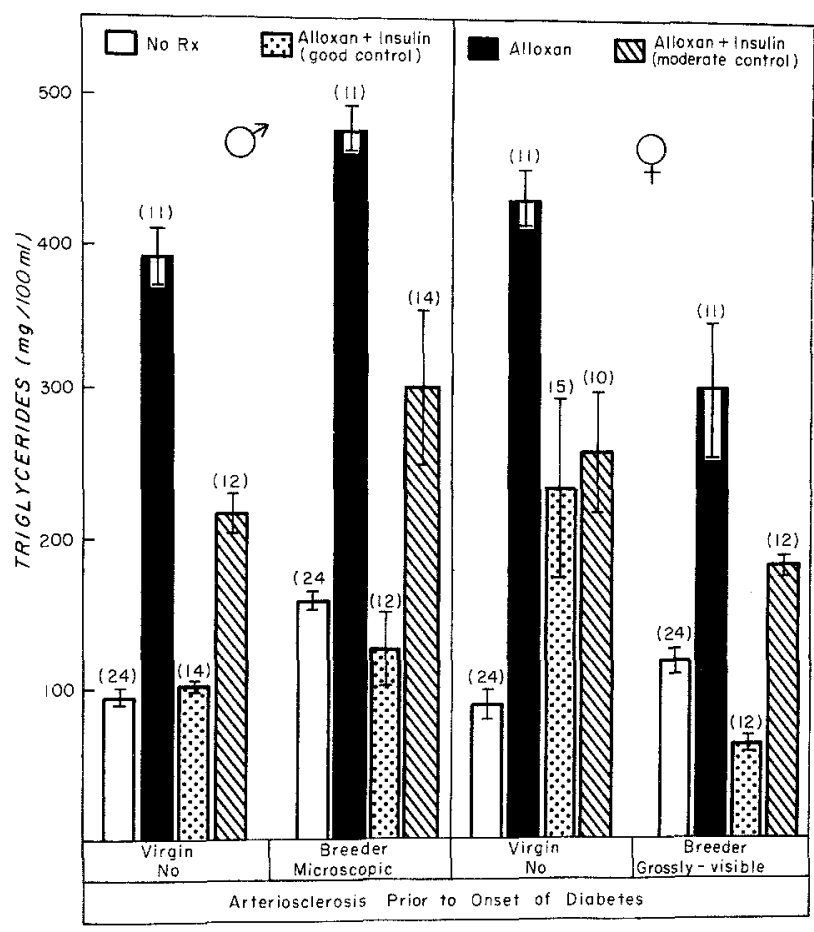

Fig. 2. Changes in circulating triglycerides. The same protocol described under Figure 1 applies except that all of the samples were taken at autopsy ( 5 months after alloxan) and values for males and females are depicted. The hight of each column indicates mean \pm SEM,()$=$ number of samples

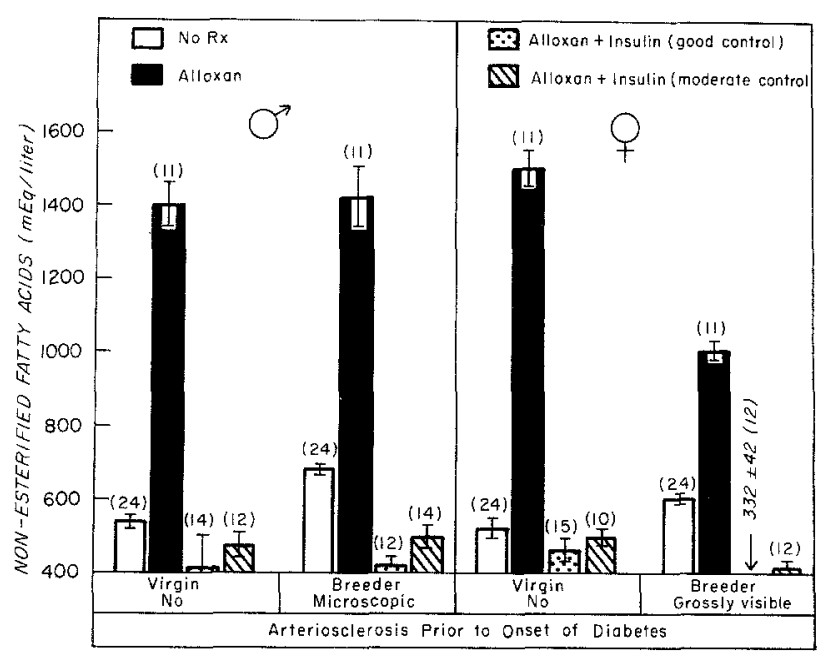

Fig. 3. Changes in circulating non-esterified fatty acids. Protocol and figure details as Figure 2

control was most effective in decreasing the abnormally elevated circulating triglyceride levels to normal. Animals with moderate control of their diabetes showed much less effective reduction of serum triglyceride levels (Fig. 2). 
Non-esterified Fatty Acids

Like circulating triglyceride levels, non-esterified fatty acids were greatly elevated in the untreated, diabetic rats (Fig. 3). Treatment with insulin, both good and moderate control, caused effective lowering of serum non-esterified fatty acid levels (Fig. 3). Insulin was most effective in decreasing nonesterified fatty acid levels in arteriosclerotic, female breeders.

\section{Serum Total Cholesterol}

Good and moderate control with insulin were both effective in reducing circulating cholesterol levels (Fig. 4).

\section{Corticosterone}

The severe diabetes caused circulating corticosterone levels to become chronically elevated (Fig. 5). Except for females provided good control of their diabetes, treatment with insulin had little or no ameliorative effects on the hyperadrenocorticism due to chronic diabetes (Fig. 5).

\section{Histopathology}

The histopathological changes induced by chronic diabetes were identical in incidence, morphology, and severity to those reported in detail in our earlier work [8] and will not be repeated here. All of the severely diabetic animals had fatty livers together with advanced pancreatitis and glomerulosclerosis. The beta cells of the islets of Langerhans were completely degranulated and the islets contracted and fibrotic. The adrenal cortices were greatly hypertrophied and extensively depleted of lipid. The thymi were severely involuted. The testes and ovaries were atrophic. None of the diabetic virgin rats provided good or moderate control were found to have any evidence of cardiovascular disease. Sixty-eight per cent of the untreated diabetic virgin rats (normally resistant to arterial disease) manifested intimal calcification of the epicardial branches of their coronary arteries (Fig. 6). In addition, their aortae contained scattered foci of early atheromatous lesions consisting of intimal accumulations of mucopolysaccharide capped-over by collagen (Fig. 7). These early lesions were devoid of lipid. Diabetic breeder rats provided good insulin control did not show any exacerbation of their pre-existing arteriosclerosis (Fig. 8). The advanced aortic sclerosis characteristic of breeder rats was greatly exacerbated in those breeder rats made severely diabetic or those given restricted insulin

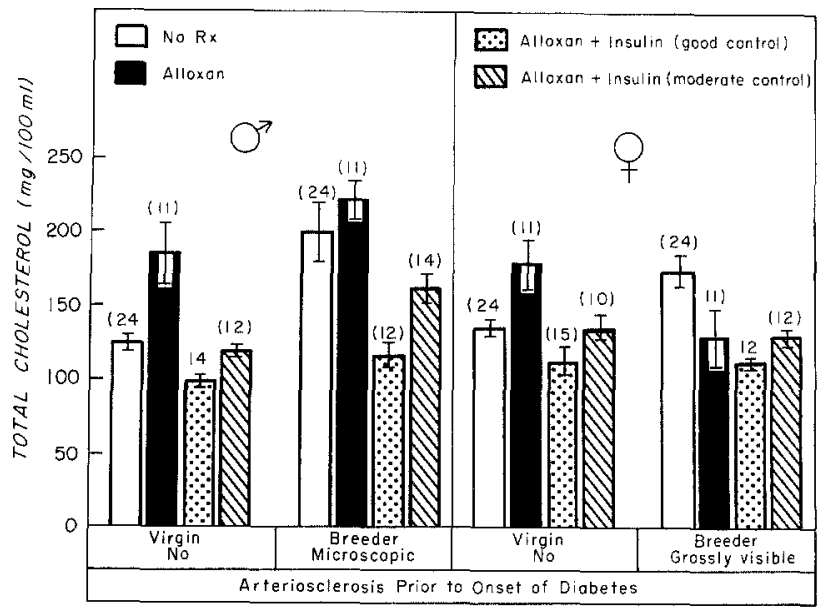

Fig. 4. Changes in circulating total cholesterol. Protocol and figure details as Figure 2

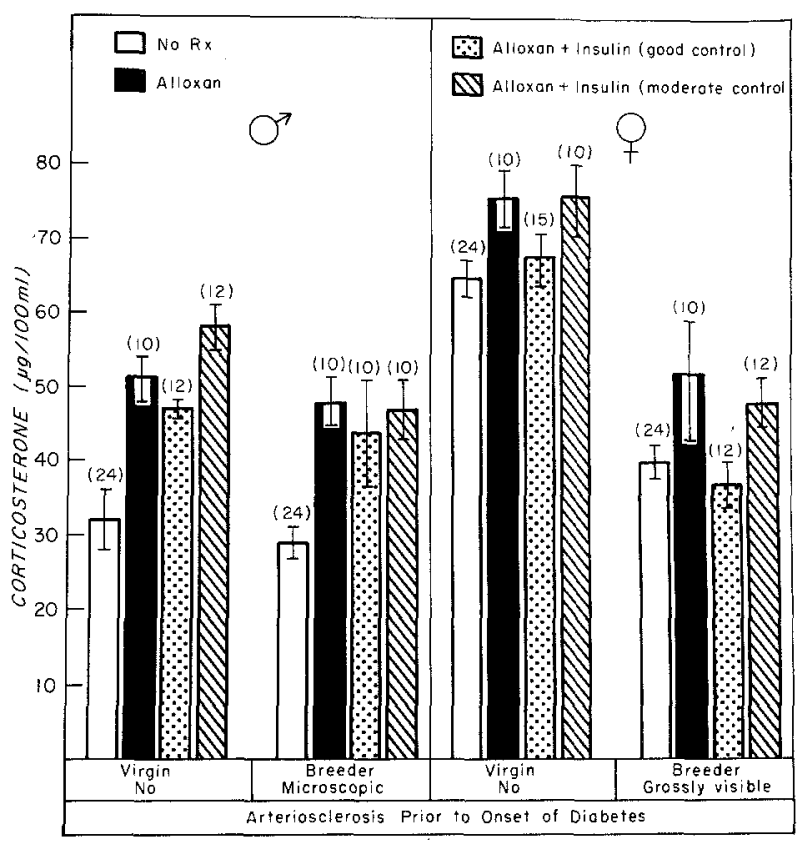

Fig. 5. Changes in circulating corticosterone. Protocol and figure details as Figure 2

therapy, with intensification of the usual severity of lesions, increased number of sclerotic foci, and intensification of the extension of the arteriosclerosis into the mesenteric, renal, and peripheral arteries. Microscopically, these advanced lesions were characterized by intense elastolytic degeneration, accumulation of mucopolysaccharide-rich ground substance, and cartilaginous metaplasia bordering on differentiation into osseous metaplasia (Fig. 9).

A summary is presented (Table 2) in an attempt to quantify the gross and microscopic lesions of the 


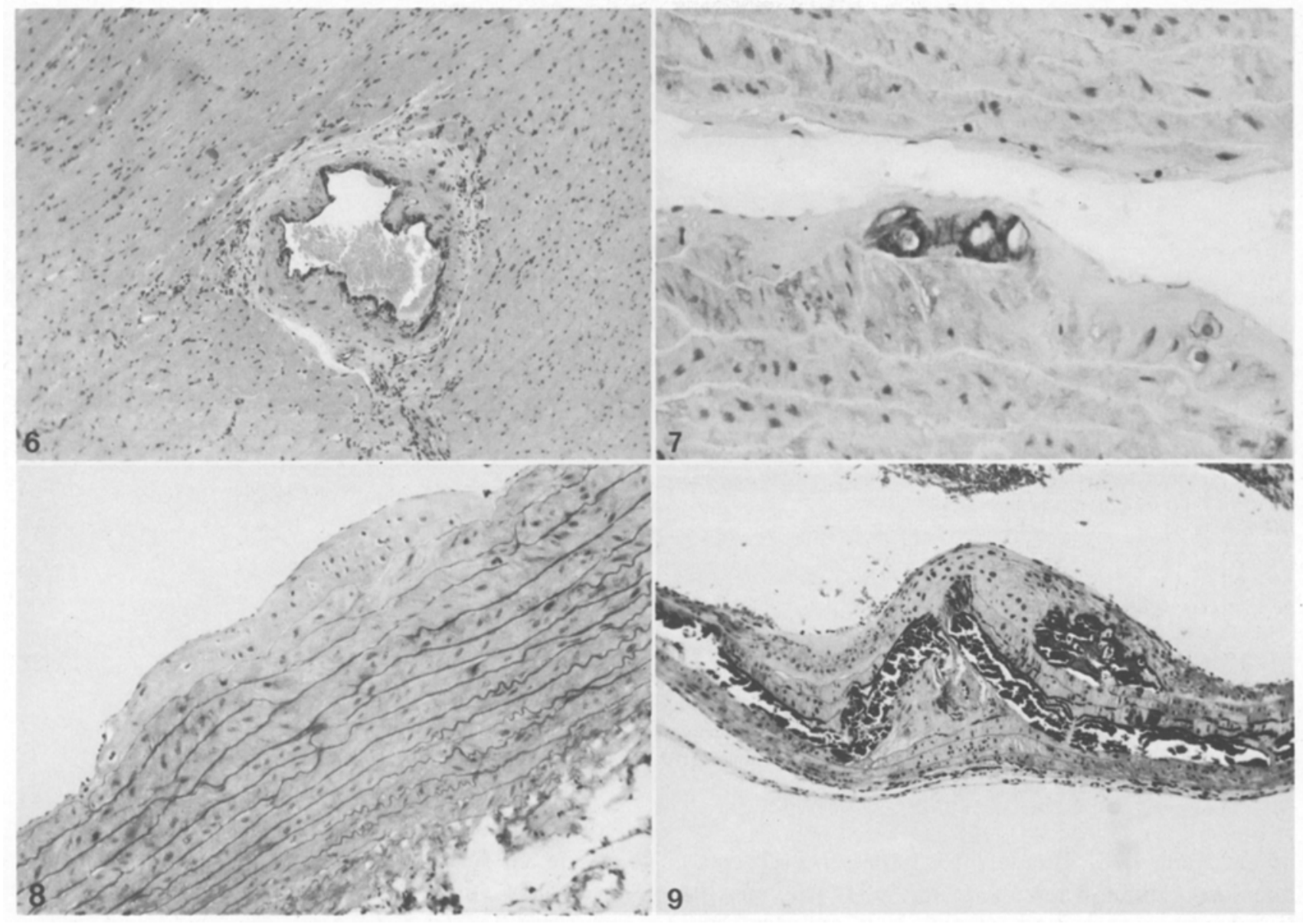

Fig. 6. Epicardial branch of a coronary artery of a normally arteriosclerosis-free, virgin male Sprague-Dawley rat subjected to 5 months of unrelenting alloxan-induced diabetes. The tunica intima contains extensive accumulations of a calcium and eosin, $\times 40$

Fig. 7. High power view of an early atheromatous lesion in the aorta of an alloxan diabetic, virgin male. The tunica intima is distended by mucoproteinaceous material (light grey). Large, rounded mesenchymal cells rich in mucopolysaccharide (black material) are seen at the center of the lesion. Rapidly differentiating mesenchymal cells (probably smooth muscle cells from the tunica media) appear at the edge
(right side of photomicrograph). Hale stain, $\times 85$

Fig. 8. Abdominal aorta of a female breeder rat with spontaneous arteriosclerosis and mild hyperglycaemia illustrating the severity of arterial disease prior to the induction of alloxan-diabetes. Early lesions are comprised of foci of endothelial hyperplasia, differentiating mesenchymal cells, and are subtended by beginning elastosis of the inner medial lamellae. The tunica intima and inner media stains strongly positive for mucopolysaccharide. Haematoxylin and eosin, $\times 80$

Fig. 9. Abdominal aorta of a female breeder rat subjected to 5 months of alloxan-induced diabetes and given moderately adjusted insulin therapy. The aorta is severely ectatic and there is extensive medial degeneration characterized by severe elastic tissue degeneration, mucopolysaccharide accumulation (black) and calcification. There is a focus of cartilaginous metaplasia central to an intimal fibroendothelial plaque. Haematoxylin and eosin, $\times 45$

treated and non-treated alloxan-diabetic rats. The induction of alloxan-diabetes in virgin rats was associated with the differentiation of mesenchymal cells into rounded, deeply basophilic, and mucopolysaccharide-rich cartilaginous cells (Fig. 7). Alloxandiabetes in breeder rats was associated with worsening elastolytic change, accumulation of mucopolysaccharide-rich ground substance, collagen, and accelerated cartilaginous metaplasia (Fig. 9).

\section{Discussion}

It is of interest that the arteriosclerotic female breeder rats manifested the greatest ameliorative effects because of insulin therapy. Breeder rats become progressively more obese with each successive breeding and have greater adipose tissue reserve. The availability of endogenous lipids for fatty acid oxidation helps to dispel the degree of insu- 
Table 2. Summary of the severity of grossly visible and microscopic aortic and coronary artery lesions in virgin and breeder, SpragueDawley rats subjected to alloxan diabetes and provided good or moderate control of their diabetes with insulin

\begin{tabular}{|c|c|c|c|c|c|c|c|c|}
\hline & \multicolumn{4}{|l|}{ Males } & \multicolumn{4}{|c|}{ Females } \\
\hline & \multirow[t]{2}{*}{$\begin{array}{l}\text { No. of } \\
\text { rats }\end{array}$} & \multirow{2}{*}{$\begin{array}{l}\text { Grossly } \\
\text { visible } \\
\text { aortic } \\
\text { sclerosis }\end{array}$} & \multicolumn{2}{|c|}{$\begin{array}{l}\text { Microscopic } \\
\text { sclerosis }\end{array}$} & \multirow[t]{2}{*}{$\begin{array}{l}\text { No. of } \\
\text { rats }\end{array}$} & \multirow{2}{*}{$\begin{array}{l}\text { Grossly } \\
\text { visible } \\
\text { aortic } \\
\text { sclerosis }\end{array}$} & \multicolumn{2}{|c|}{$\begin{array}{l}\text { Microscopic } \\
\text { sclerosis }\end{array}$} \\
\hline & & & aortic & coronary & & & aortic & coronary \\
\hline \multicolumn{9}{|l|}{ Virgins } \\
\hline Controls - no treatment & 24 & 0 & 0 & 0 & 24 & 0 & 0 & 0 \\
\hline Alloxan alone & 11 & 0 & $t^{a}$ & $+t^{\mathrm{a}}$ & 11 & 0 & $t^{a}$ & $+t^{a}$ \\
\hline $\begin{array}{l}\text { Alloxan + Insulin } \\
\text { Good control }\end{array}$ & 18 & 0 & 0 & 0 & 20 & 0 & 0 & 0 \\
\hline $\begin{array}{l}\text { Alloxan + Insulin } \\
\text { Moderate control }\end{array}$ & 13 & 0 & 0 & 0 & 18 & 0 & 0 & 0 \\
\hline \multicolumn{9}{|l|}{ Breeders } \\
\hline Controls - no treatment & 24 & + & + & + & 24 & ++ & $+t$ & ++ \\
\hline Alloxan alone & 11 & + & ++ & ++ & 11 & +++ & +++ & +++ \\
\hline $\begin{array}{l}\text { Alloxan + Insulin } \\
\text { Good control }\end{array}$ & 16 & 0 & $t^{b}$ & $+^{\mathrm{b}}$ & 14 & $t^{c}$ & $t^{c}$ & $t^{c}$ \\
\hline $\begin{array}{l}\text { Alloxan + Insulin } \\
\text { Moderate control }\end{array}$ & 15 & + & ++ & ++ & 16 & +++ & +++ & +++ \\
\hline
\end{tabular}

Scoring system: $+=$ minimal severity $++=$ moderate severity $+++=$ severe

${ }^{a} 67 \%$ of the males and $69 \%$ of the females displayed lesions; the remainder were free of arterial disease

b $76 \%$ of the male breeders displayed lesions of minimal severity; $24 \%$ displayed aortic and coronary lesions of moderate severity

${ }^{\circ} 84 \%$ of the female breeders displayed lesions of minimal severity; $16 \%$ displayed aortic and coronary lesions of severe degree

lin deficiency [12]. It is intriguing that obese breeder rats lost weight when made diabetic, whereas the lean virgin rats gained weight. It is apparent that insulin treatment was adipokinetic in both the diabetic breeder and virgin rats. Of special importance is the fact that our twice-daily measurement of body weight and testing urine for glucose and protein to gauge the severity of diabetes was deceptive. That is, despite good control of the diabetes based on reduced urinary glucose levels, blood levels of glucose remained elevated. It is also of interest that insulin therapy was not as effective in controlling blood glucose as it was in controlling blood lipids.

We [6-9] and others [13] have found that alloxandiabetic rats manifest much greater increases in triglycerides and non-esterified fatty acids than cholesterol. It is believed that the hypertriglyceridaemia of the diabetic rat is due to defective removal of the very low density lipoprotein triglycerides and diminished activity of hepatic triglyceride lipase and adipose tissue lipoprotein lipase at the site of serum triglyceride hydrolysis [13]. The ubiquitous occurrence of hepatic lipidosis, even in animals treated with insulin, would attest to the central role of the liver. Lipid removal may be just as important as lipid synthesis. The apparent superior regulation of lipid metabolism over carbohydrate metabolism by insulin in these animals parallels the long recognized clinical experience that control of blood glucose does not necessarily control abnormalities in lipid metabolism $[13,14]$.

Concomitant with thymus gland involution, the adrenal hypertrophy observed in these diabetic rats is suggestive of increased pituitary gland secretion of corticotrophin (ACTH). Diabetes is a metabolic stress which causes ACTH release; insulin therapy prevents adrenal hypertrophy in the alloxan diabetic rat [15]. Insulin deficient, alloxan-diabetic rats respond to exogenous $\mathrm{ACTH}$ stimulation with significantly higher plasma corticosterone levels than do insulin-treated rats $[16,17]$. Our earlier findings that alloxan-induced diabetes is a severely stressful condition [6-9] and our present report concurs with all of the foregoing [15-17]. It is of interest that insulin therapy did not cause any significant amelioration of the adrenocortical duress of diabetes in our animals.

The most salient feature of this investigation is the appearance of arterial disease in sclerosis free virgin rats made diabetic, and the prevention of the extension of the pre-existent arteriosclerosis of diabetic breeder rats provided good control. It is of interest that the diabetic virgin rats should manifest such a high incidence $(68 \%)$ and predilection for intimal calcification of the epicardial branches of their coronary arteries, and aortic lesions consisting of mucopolysaccharide and collagen accumulation with 
little or no lipid infiltrate. The absence of lipid in these lesions is all the more remarkable in view of the obesity, fatty liver, and severe hyperlipidaemia in these untreated, diabetic, virgin rats. Although insulin therapy protected these virgin rats against the development of aortic and coronary artery lesions, glomerulosclerosis and renal tubular degeneration appeared inexorably irrespective of treatment. Only the diabetic breeder rats with pre-existent arteriosclerosis that were provided good control of their diabetes were afforded protection against the advanced arteriosclerosis which attends untreated or moderately controlled diabetes, that is advanced ground substance degenerative changes in the intima and media, elastosis, and calcification of the aorta, coronary, carotid, renal, mesenteric and peripheral arteries [18-20].

Acknowledgements. This work was supported by grants from the National Heart, Lung, and Blood Disease Institute (HL-21418), the National Institute on Ageing (AG-585), and the Southwestern Ohio Heart Association.

The authors are grateful for the dedication and expertise of D. Conatser, E. Domingo, W. Goodhew, and J. Wexler.

\section{References}

1. Wexler BC (1964) Spontaneous arteriosclerosis in repeatedly bred male and female rats. J Atheroscler Res 4: 57-80

2. Wexler BC, Fischer CW (1963) Hyperplasia of the islets of Langerhans in arteriosclerotic rats. Nature 200: 33-37

3. Wexler BC, Fischer CW (1963) Abnormal glucose tolerance in repeatedly bred rats with arteriosclerosis. Nature 200: 133-136

4. Wexler BC (1970) Arteriosclerosis of the pancreas and changes in the islets of Langerhans of repeatedly bred rats. $\mathrm{Br}$ J Exp Pathol 51: 107-113

5. Wexler BC (1979) Spontaneous arteriosclerosis in old, male, virgin Sprague-Dawley rats. Atherosclerosis 34: 277-290

6. Wexler BC, Saroff J, Judd JT (1970) Variations in response to severe alloxan diabetes in arteriosclerotic and nonarteriosclerotic rats. Diabetes 19: 311-323
7. Wexler BC (1970) Histopathologic responses to severe alloxan diabetes in arteriosclerotic and nonarteriosclerotic rats. Diabetes 19: 324-336

8. Wexler BC (1971) Pathophysiologic responses to severe chronic alloxan diabetes in arteriosclerotic and nonarteriosclerotic rats. Atherosclerosis 14: 289-307

9. Wexler BC (1976) Effects of hypophysectomy on alloxandiabetic, arteriosclerotic, breeder vs nonarteriosclerotic, virgin rats. Atherosclerosis 25: $13-30$

10. Guillemin R, Clayton GW, Lipscombe HS, Smith JD (1959) Fluorometric measurement of rat plasma and adrenal corticosterone concentration. J Lab Clin Med 53: 830-832

11. Snedecor GW, Cochran WG (1967) In: Statistical methods, 6th edn. Iowa State University Press, Ames

12. Steiner DF, Rauda V, Williams RH (1961) Severe ketoacidosis in the alloxan diabetic rat. Endocrinology 68: 809-817

13. Hennes AR, Redding TW (1961) Defective synthesis of triglyceride fatty acids from 1-C-14 acetate in the well-controlled stable adult diabetic. Diabetes 10: 85-89

14. Bierman EL, Dole VP, Roberts TN (1957) An abvormality of non-esterified fatty acid metabolism in diabetes mellitus. Diabetes 6: $475-479$

15. Field JB (1953) Prevention of adrenal cortical hypertrophy in diabetic rats by the use of insulin. Endocrinology 56: 499-506

16. L'Age M, Langholz J, Fechner W, Salzmann H (1974) Disturbances of the hypothalamo-hypophysial-adrenocortical system in the alloxan diabetic rats. Endocrinology 95: 760-765

17. Wexler BC, Lutmer RF (1975) Adrenal glandular lipids and circulating corticosterone in severely diabetic rats. Br J Exp Pathol 56: 299-306

18. Wexler BC, True CW (1963) Carotid and cerebral arteriosclerosis in the rat. Circ Res 12: 659-666

19. Wexler BC (1964) Spontaneous coronary arteriosclerosis in repeatedly bred male and female rats. Circ Res 14: 32-48

20. Wexler BC (1964) Spontaneous arteriosclerosis of the mesenteric, renal and peripheral arteries of repeatedly bred rats. Circ Res 15: 485-496

Received: October 12, 1979, and in revised form: April 11, 1980

Dr. Bernard C. Wexler, Director

The May Institute for Medical Research

of the Jewish Hospital Association

421 Ridgeway Ave

Cincinnati, OH 45229

USA 